\title{
Variablendefinition in fallbezogenen Krankenhausabrechnungsdaten - Fallstricke und Lösungsmöglichkeiten
}

\section{Definition of Variables in Hospital Discharge Data: Pitfalls and Proposed Solutions}

\section{()(1) $\odot \Theta$}

\section{Autoren \\ Ulrike Nimptsch', 2, Melissa Spoden ${ }^{1,2}$, Thomas Mansky}

\section{Institute}

1 Fachgebiet Strukturentwicklung und Qualitätsmanagement im Gesundheitswesen, Technische Universität Berlin, Berlin

2 Fachgebiet Management im Gesundheitswesen, Technische Universität Berlin, Berlin

\section{Schlüsselwörter \\ Fallpauschalenbezogene Krankenhausstatistik (DRG-Statis- tik), Krankenhausabrechnungsdaten, Variablendefinition, Analyseeinheiten, Risikoadjustierung}

\section{Key words}

DRG Statistics, hospital discharge data, definition of variables, unit of analysis, risk adjustment

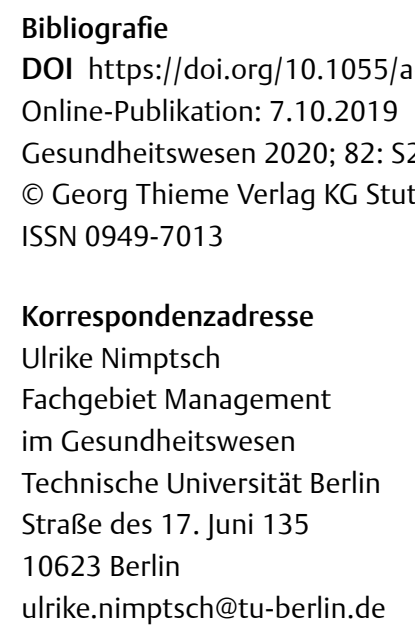

\section{ZUSAMMENFASSUNG}

In Deutschland ist mit der Fallpauschalenbezogenen Krankenhausstatistik (DRG-Statistik) eine Vollerhebung der Abrechnungsdaten nahezu aller akutstationären Krankenhausbehandlungsfälle verfügbar. Die Mikrodaten, d. h. die Datensätze der einzelnen Behandlungsfälle, werden von den Forschungs- datenzentren der Statistischen Ämter des Bundes und der Länder für die wissenschaftliche Nutzung bereitgestellt. Krankenhausabrechnungsdaten werden zu Abrechnungszwecken erhoben und haben - wie andere Datenquellen auch - spezifische Besonderheiten und Eigenschaften, die bei der Planung von Analysen und im Studiendesign angemessen berücksichtigt werden müssen. Eine besondere Herausforderung ist dabei die sachgerechte Operationalisierung von Analyseeinheiten, Zielgrößen und weiteren Variablen, die u. a. eine mögliche unterschiedliche Kodierpraxis in verschiedenen Krankenhäusern antizipiert und deren Auswirkungen über eine durchdachte Variablendefinition minimiert. In diesem Beitrag wird anhand von praktischen Beispielen aufgezeigt, was bei der Definition von Variablen in fallbezogenen Krankenhausabrechnungsdaten beachtet werden sollte, um das Risiko von kodierungs- oder anderweitig bedingten Verzerrungen möglichst gering zu halten. Ausgehend von den Erfahrungen der Autoren werden die wichtigsten Schritte und Herausforderungen in Bezug auf ein typisches retrospektives Beobachtungsstudiendesign in der Versorgungsforschung beschrieben. Basierend auf bereits durchgeführten Projekten auf der Grundlage der Mikrodaten der Fallpauschalenbezogenen Krankenhausstatistik (DRGStatistik) werden Beispiele anhand eigener Berechnungen illustriert. Die Verlässlichkeit und Interpretierbarkeit von Analysen auf der Grundlage der Mikrodaten der DRG-Statistik hängt entscheidend von der sachgerechten Operationalisierung der Variablen ab. Um das Risiko von Verzerrungen und Fehlinterpretationen möglichst gering zu halten, sind umfangreiche Vorüberlegungen notwendig, die neben medizinisch-klinischen Aspekten auch den Kontext der Datenerhebung und die klassifikatorischen Möglichkeiten zur Abbildung bestimmter Sachverhalte berücksichtigen. Es ist zu hoffen, dass die Akzeptanz von Studien auf der Grundlage von Krankenhausabrechnungsdaten weiter zunimmt und der wertvolle Datenbestand der DRG-Statistik in Zukunft noch intensiver für Forschungszwecke genutzt wird. 


\begin{abstract}
In Germany, the Diagnosis-Related Group Statistics (DRG Statistics) supply full coverage of inpatient episodes in acute care hospitals. The Research Data Centres of the Federal Statistical Office and the Statistical Offices of the Federal States provide the microdata of the DRG Statistics, namely hospital discharge files of each inpatient case, for scientific research. Hospital discharge data are generated for administrative purposes. As well as other data sources, they have specific features and characteristics, which should be considered in planning and designing research studies. A key challenge is the appropriate and sophisticated operationalization of units of analysis, targets variables, and other study variables. The methodological approach should consider, among other factors, differing coding behaviour between hospitals in order to minimize the risk of bias. This contribution shows by practical examples what should be incorporated in variable definition to ensure that the risk of bias by coding beha-
\end{abstract}

viour or other factors is minimized to the greatest possible degree. First of all, the features and characteristics of the German hospital discharge data are outlined. Based on the authors' experiences, basic steps and challenges in observational health services research studies are described. Examples are illustrated by our own calculations, derived from previous studies based on the microdata of the DRG Statistics. The reliability and validity of analyses based on hospital discharge data are crucially dependent on the appropriateness of variable definition. To minimize the risk of bias and misinterpretation, extensive preliminary considerations are required which involve clinical aspects, as well as the context of data collection and technical classification opportunities. Hopefully, there will be greater acceptance of research based on hospital discharge data, so that these valuable data will be used more frequently for research purposes in the future.

\section{Hintergrund und Ziel}

Fallbezogene Krankenhausabrechnungsdaten sind eine wichtige Datenquelle für die Gesundheits- und Versorgungsforschung. Mit der Fallpauschalenbezogenen Krankenhausstatistik (DRG-Statistik) ist in Deutschland eine Vollerhebung der Abrechnungsdaten der akutstationären Krankenhausbehandlungsfälle verfügbar. Die Mikrodaten, d. h. die Datensätze der einzelnen Behandlungsfälle, werden von den Forschungsdatenzentren der Statistischen Ämter des Bundes und der Länder für die wissenschaftliche Nutzung bereitgestellt. In den letzten Jahren wurden diese Daten zunehmend genutzt, um die Strukturen der akutstationären Krankenhausversorgung zu beschreiben, zeitliche Entwicklungen darzustellen und mögliche Handlungsfelder zur Verbesserung der Versorgung zu identifizieren.

Krankenhausabrechnungsdaten werden zu Abrechnungszwecken erhoben und haben - wie andere Datenquellen auch - spezifische Besonderheiten und Eigenschaften, die bei der Planung von Analysen und im Studiendesign berücksichtigt werden müssen. Eine besondere Herausforderung ist dabei die sachgerechte Operationalisierung von Analyseeinheiten, Zielgrößen und weiteren Variablen, die u. a. eine mögliche unterschiedliche Kodierpraxis in verschiedenen Krankenhäusern antizipiert und deren Auswirkungen über eine durchdachte Variablendefinition minimiert.

In diesem Beitrag wird anhand von praktischen Beispielen aufgezeigt, was bei der Analyse fallbezogener Krankenhausabrechnungsdaten beachtet werden sollte, um das Risiko von kodierungs- oder anderweitig bedingten Verzerrungen möglichst gering zu halten. Ausgehend von Erfahrungen der Autoren werden die wichtigsten Schritte und Herausforderungen in Bezug auf ein typisches retrospektives Beobachtungsstudiendesign in der Versorgungsforschung beschrieben. Dabei wird insbesondere auf mögliche Fallstricke und Fehlerquellen eingegangen. Basierend auf bereits durchgeführten Projekten auf der Grundlage der Mikrodaten der Fallpauschalenbezogenen Krankenhausstatistik (DRG-Statistik), die vom Forschungsdatenzentrum des Statistischen Bundesamtes bereitgestellt wurden, werden Beispiele anhand eigener Berechnungen illustriert.

\section{Eigenschaften von Krankenhausabrechnungsdaten}

In den Jahren 2003 und 2004 wurde in Deutschland zur Vergütung der akutstationären Krankenhausleistungen (mit Ausnahme der Psychiatrie und Psychosomatik) das fallpauschalierende Vergütungssystem der Diagnosis Related Groups (DRG) eingeführt. In diesem Vergütungssystem wird ein Krankenhausbehandlungsfall auf der Grundlage von Diagnosen, Prozeduren, administrativen und demografischen Merkmalen und weiteren Informationen in eine Fallpauschale eingruppiert. Die Eingruppierung bestimmt die Höhe des Entgeltes für den Behandlungsfall. Dies hat zur Folge, dass die genannten Informationen Bestandteil der Rechnung für einen Behandlungsfall sind und von allen Krankenhäusern, die dem DRGEntgeltbereich unterliegen, standardisiert elektronisch erfasst werden müssen. Die Datenerhebung in den Krankenhäusern dient damit primär der Abwicklung des elektronischen Rechnungs- und Zahlungsverkehrs zwischen Krankenhäusern und gesetzlichen oder privaten Krankenkassen.

Für die Erfassung der Diagnosen und Prozeduren gelten einheitliche Kodierrichtlinien, die eine vergleichbare Dokumentation gewährleisten sollen [1]. Daneben werden die entgeltrelevanten Inhalte von Krankenhausabrechnungsdaten mehrfach geprüft. Im Vorfeld der Rechnungslegung prüft in der Regel das krankenhausinterne Medizincontrolling, das für das Erlösmanagement im Krankenhaus verantwortlich ist, die Vollständigkeit und Richtigkeit der entgeltrelevanten Dokumentationsinhalte. Nachdem die Rechnung übermittelt wurde prüfen die Krankenkassen die Datensätze auf ihre Plausibilität. In Verdachtsfällen werden gesonderte Prüfungen, z. B. durch den Medizinischen Dienst der Krankenkassen (MDK), anhand von Informationen aus der Krankenakte durchgeführt. Im Jahr 2017 wurden mehr als 14\% der stationären Krankenhausabrechnungen einer Einzelfallprüfung durch den Medizinischen Dienst der Krankenkassen unterzogen. Dabei wurde ca. jede zweite Rechnung korrigiert [2]. Damit handelt es sich bei Krankenhausabrechnungsdaten - entgegen weitverbreiteter Annahmen - um einen (zumindest in Bezug auf entgeltrelevante Inhalte) vergleichsweise gut geprüften Datensatz. 
Die Abrechnungsdaten aller Krankenhäuser im Anwendungsbereich des $\S 1$ des Krankenhausentgeltgesetzes (KHEntgG), d. h. im Geltungsbereich des DRG-Systems, werden an einer zentralen Stelle zusammengeführt: Einmal jährlich übermitteln die Krankenhäuser ihre vollständigen fallbezogenen Abrechnungsdaten in einem festgelegten Format (gemäß § $21 \mathrm{KHEntgG)} \mathrm{an} \mathrm{das} \mathrm{Institut} \mathrm{für} \mathrm{das}$ Entgeltsystem im Krankenhaus (InEK), das mit der Einführung, Weiterentwicklung und Pflege des DRG-Vergütungssystems beauftragt ist. Die Übermittlung erfolgt zum 31. März für das jeweils vorangegangene Kalenderjahr, sodass eventuelle Korrekturen größtenteils im Datenbestand berücksichtigt sein dürften.

Mit diesem Datenbestand verfügt Deutschland über ein nahezu vollständiges Register der akutstationären Krankenhausbehandlungen in der somatischen Versorgung [3]. Ein ausgewähltes Merkmalsspektrum dieser Daten wird vom InEK an das Statistische Bundesamt zur Erstellung von Bundesstatistiken weitergeleitet. Von den Forschungsdatenzentren der Statistischen Ämter des Bundes und der Länder werden die Mikrodaten der sogenannten DRG-Statistik ab dem Datenjahr 2005 für wissenschaftliche Auswertungen zur Verfügung gestellt [4].

Die Bezugsgröße in den Mikrodaten der DRG-Statistik ist der Krankenhausbehandlungsfall, also eine Behandlungsepisode von der Aufnahme bis zur Entlassung eines Patienten, für die, entsprechend der Abrechnungsbestimmungen der Fallpauschalenverordnung (FPV, siehe [5]), eine Rechnung gestellt wird. Da in den zur Auswertung verfügbaren Mikrodaten kein Personenkennzeichen enthalten ist, können mehrere Krankenhausaufenthalte einer Person, bspw. bei einer Verlegung von einem Krankenhaus in ein anderes, nicht miteinander verknüpft werden.

Die Daten enthalten zu jedem Behandlungsfall neben demografischen Angaben (z. B. Alter bei Aufnahme, Geschlecht, Kreiskennzeichen des Patientenwohnortes) verschiedene administrative Informationen. Diese umfassen u. a. den Aufnahmeanlass (z. B. Einweisung, Notfall, Verlegung aus einem anderen Krankenhaus) und die Entlassungsart (z. B. regulär entlassen, verlegt oder verstorben), die Art der behandelnden Fachabteilung/-en und die Anzahl der Belegungstage. Haupt- und Nebendiagnosen sind nach der International Classification of Diseases, German Modification (ICD-10GM), Prozeduren nach dem Operationen- und Prozedurenschlüssel (OPS) kodiert. Daneben sind bestimmte weitere medizinische Angaben enthalten, die zur Ermittlung der DRG-Fallpauschale notwendig sind (Anzahl der Stunden maschineller Beatmung, Aufnahmegewicht bei Neugeborenen). Das behandelnde Krankenhaus lässt sich über das anonymisierte Institutionskennzeichen abgrenzen, sodass krankenhausbezogene Auswertungen prinzipiell möglich sind. Da Regionalinformationen, sowohl zum Krankenhausstandort als auch zum Patientenwohnort, enthalten sind, können die DRG-Daten auch für regionale Analysen genutzt werden [6].

Da die Daten der DRG-Statistik eine Vollerhebung der Behandlungsfälle in Krankenhäusern im Anwendungsbereich des $\S 1$ KHEntgG darstellen, sind sie in Bezug auf die meisten Krankheitsbilder bzw. Behandlungen in der akutstationären somatischen Versorgung als nahezu vollständig zu werten. Abgesehen von dem Bereich der Psychiatrie und Psychosomatik, sowie wenigen weiteren Ausnahmen (z. B. Behandlungen zu Lasten der gesetzlichen Unfallversicherung in Krankenhäusern der Berufsgenossenschaften) [7] wird jeder Behandlungsfall - unabhängig von der Art der Versiche- rung des Patienten - erfasst. Beachtet werden muss jedoch, dass diese Daten ausschließlich die vollstationäre Versorgung abbilden und ambulante Krankenhausbehandlungen nicht enthalten sind. Deshalb eignen sich die Mikrodaten der DRG-Statistik insbesondere zur Untersuchung von Versorgungsleistungen, die vorwiegend oder ausschließlich stationär durchgeführt werden, wie die Behandlung schwerer akuter Erkrankungen (z. B. Herzinfarkt oder Schlaganfall) oder größere Operationen [8].

\section{Definition der Analyseeinheiten: Wie kann die interessierende Untersuchungsgruppe abgebildet werden?}

Einer der ersten und wichtigsten Schritte bei der Planung von Beobachtungsstudien auf der Grundlage der Mikrodaten der DRG-Statistik ist die Frage, welche Einheiten betrachtet werden sollen. In der Regel beziehen sich Fragestellungen auf Behandlungsfälle mit bestimmten Merkmalen. Solche Falldefinitionen lassen sich anhand von beliebigen Merkmalskombinationen aus Diagnosen, Prozeduren und weiteren Angaben definieren. Die Herausforderung dabei besteht darin, die Analyseeinheiten anhand der verfügbaren Informationen so zu spezifizieren, dass sich die Interpretation der Ergebnisse auf eine klar abgrenzbare Gruppe von Behandlungsfällen beziehen kann.

\section{Definition der Analyseeinheiten ausgehend von einem Krankheitsereignis}

In der Versorgungsforschung ist es häufig von Interesse, wie viele Patienten mit einer bestimmten Erkrankung im Krankenhaus behandelt werden. Die stationären Behandlungsfälle im Zusammenhang mit einer bestimmten Erkrankung lassen sich in den Mikrodaten der DRG-Statistik anhand der dokumentierten Diagnosen identifizieren.

Für eine sachgerechte Operationalisierung sind gute Kenntnisse der Systematik der ICD-10-GM erforderlich. In manchen Fällen ist die interessierende Erkrankung durch mehrere ICD-Kodes abbildbar, die ggf. in verschiedenen Kapiteln gelistet sind. Ein Beispiel dafür ist die Stenose oder Insuffizienz der Aortenklappe, die in der ICD-10-GM - je nach Ätiologie - unter „Sonstige Formen der Herzkrankheit“ (I35.-), unter „Chronische rheumatische Herzkrankheiten“ (I06.-), sowie unter „Angeborene Fehlbildungen des Kreislaufsystems“ (Q23.0, Q23.1) verzeichnet ist. Je nach Fragestellung muss entschieden werden, welche dieser Kodes bei der Definition der Analyseeinheiten einbezogen werden sollen. Grundsätzlich sind die Möglichkeiten zur Identifizierung und Differenzierung von Erkrankungen durch den Detaillierungsgrad der ICD-10 begrenzt. Weitergehende Klassifikationen wie bspw. die Internationale Klassifikation der Krankheiten für die Onkologie (ICD-O) kommen in der Krankenhausabrechnung nicht zur Anwendung und sind damit auch nicht in den Daten enthalten.

Bei der Definition der Analyseeinheiten anhand von Diagnosen müssen die unterschiedlichen Bedeutungen von Haupt- und $\mathrm{Ne}$ bendiagnosen im deutschen DRG-System beachtet werden. Die Hauptdiagnose wird für jeden Behandlungsfall genau einmal vergeben. In den Kodierrichtlinien ist die Hauptdiagnose definiert als „die Diagnose, die nach Analyse als diejenige festgestellt wurde, die hauptsächlich für die Veranlassung des stationären Krankenhausaufenthaltes des Patienten verantwortlich ist“ [1]. Daneben können mehrere Nebendiagnosen zu einem Behandlungsfall dokumentiert sein. Gemäß den Kodierrichtlinien gilt als Nebendiag- 
nose „eine Krankheit oder Beschwerde, die entweder gleichzeitig mit der Hauptdiagnose besteht oder sich während des Krankenhausaufenthaltes entwickelt" [1].

Der Einschluss von Behandlungsfällen mit einer bestimmten Hauptdiagnose führt dazu, dass die Analyseeinheiten Patienten erfassen, die aufgrund der interessierenden Erkrankung im Krankenhaus behandelt wurden. Der Einschluss von Behandlungsfällen mit einer bestimmten Nebendiagnose würde dagegen Patienten abbilden, bei denen im Rahmen eines Krankenhausaufenthaltes aus anderem Anlass die interessierende Erkrankung dokumentiert wurde. Bei Nebendiagnosen ist aus den Daten zunächst auch nicht ersichtlich, ob die Erkrankung zum Zeitpunkt der Krankenhausaufnahme bereits bestand, oder sich erst im Verlauf der Behandlung entwickelt hat.

Der Unterschied zwischen Haupt- und Nebendiagnose lässt sich an einem Beispiel veranschaulichen: Die Sepsis ist ein schweres Krankheitsbild, das in der Regel stationär behandelt werden muss. Eine Sepsis kann außerhalb des Krankenhauses entstehen und damit den Krankenhausaufenthalt verursachen. Die Sepsis wäre in einem solchen Fall die Hauptdiagnose. Es kann sich jedoch auch während einer Krankenhausbehandlung aus anderem Behandlungsanlass eine Sepsis entwickeln, z. B. als Folge einer Infektion im Rahmen eines operativen Eingriffes. In einem solchen Fall wäre die Sepsis, die hier eine Komplikation darstellen würde, als Nebendiagnose dokumentiert. Behandlungsfälle mit Hauptdiagnose Sepsis unterscheiden sich in ihrem Risikoprofil von Behandlungsfällen, bei denen die Sepsis nur als Nebendiagnose kodiert ist (siehe - Abb. 1 [9]). Je nach Fragestellung kann entweder die eine oder die andere Patientengruppe von Interesse sein.

Die Anzahl von Krankenhausbehandlungsfällen mit bestimmten Hauptdiagnosen lässt auf die Anzahl stationär behandlungsbedürftiger Krankheitsereignisse in der Bevölkerung schließen. Dabei muss jedoch berücksichtigt werden, dass in den Mikrodaten der DRG-Statistik mehrfache Krankenhausaufenthalte einer Person nicht identifiziert werden können. Daher kann die Anzahl der Behandlungsfälle durch Verlegungen zwischen Krankenhäusern ver- zerrt sein. Beispielsweise werden Behandlungsfälle mit akutem Schlaganfall in ca. 15 \% aller Fälle von einem Akutkrankenhaus in ein anderes verlegt (G-IQI Indikator A.27 [9]). Dies sind zum einen sogenannte Aufwärtsverlegungen, bei denen Patienten, die zunächst in Krankenhäusern der Grund- und Regelversorgung aufgenommen wurden, meist zeitnah in spezialisierte Krankenhäuser verlegt werden, die über die erforderliche Ausstattung für spezifische Interventionen (z. B. Neuroradiologie) verfügen. Zum anderen gibt es auch Verlegungen im Anschluss an die Akutbehandlung, meist zur Durchführung der neurologischen Frührehabilitation, die in einigen Bundesländern im akutstationären Bereich erbracht wird. In beiden Fällen wird sowohl im verlegenden als auch im aufnehmenden Krankenhaus die Hauptdiagnose „akuter Schlaganfall“ vergeben, die nach den deutschen Kodierrichtlinien als Hauptdiagnose kodiert werden kann, solange der Patient eine fortgesetzte Behandlung des akuten Schlaganfalls und der unmittelbaren Folgen erhält [1]. Eine ungefilterte Zählung der schlaganfallbedingten Krankenhausbehandlungen über die Hauptdiagnose würde deshalb dazu führen, dass die Anzahl der Schlaganfälle aufgrund verlegungsbedingter Mehrfachzählungen überschätzt wird. Diese Verzerrung kann vermindert werden, indem Behandlungsfälle, die aus einem anderen Krankenhaus zuverlegt wurden, ausgeschlossen werden. Auf diese Weise werden dann jedoch nur die Fälle im erstbehandelnden Krankenhaus betrachtet, d. h. die Aussagen können sich auch nur auf die Erstversorgung beziehen [10]. Dies eine mögliche Herangehensweise. Je nach Fragestellung und betrachtetem Krankheitsereignis muss entschieden werden, wie zuverlegte oder wegverlegte Behandlungsfälle in der Definition der Analyseeinheiten berücksichtigt werden.

Zeitliche Betrachtungen von Behandlungsfallzahlen können Hinweise auf epidemiologische Entwicklungen und den zukünftigen Versorgungsbedarf liefern. Bei der Ergebnisinterpretation müssen jedoch auch Faktoren beachtet werden, die neben der interessierenden demografischen oder epidemiologischen Entwicklung die Behandlungsfallzahlen beeinflussen können. Dies können bspw. Änderungen der ICD-10-GM sein, wenn im Zeitverlauf Kodes hin-

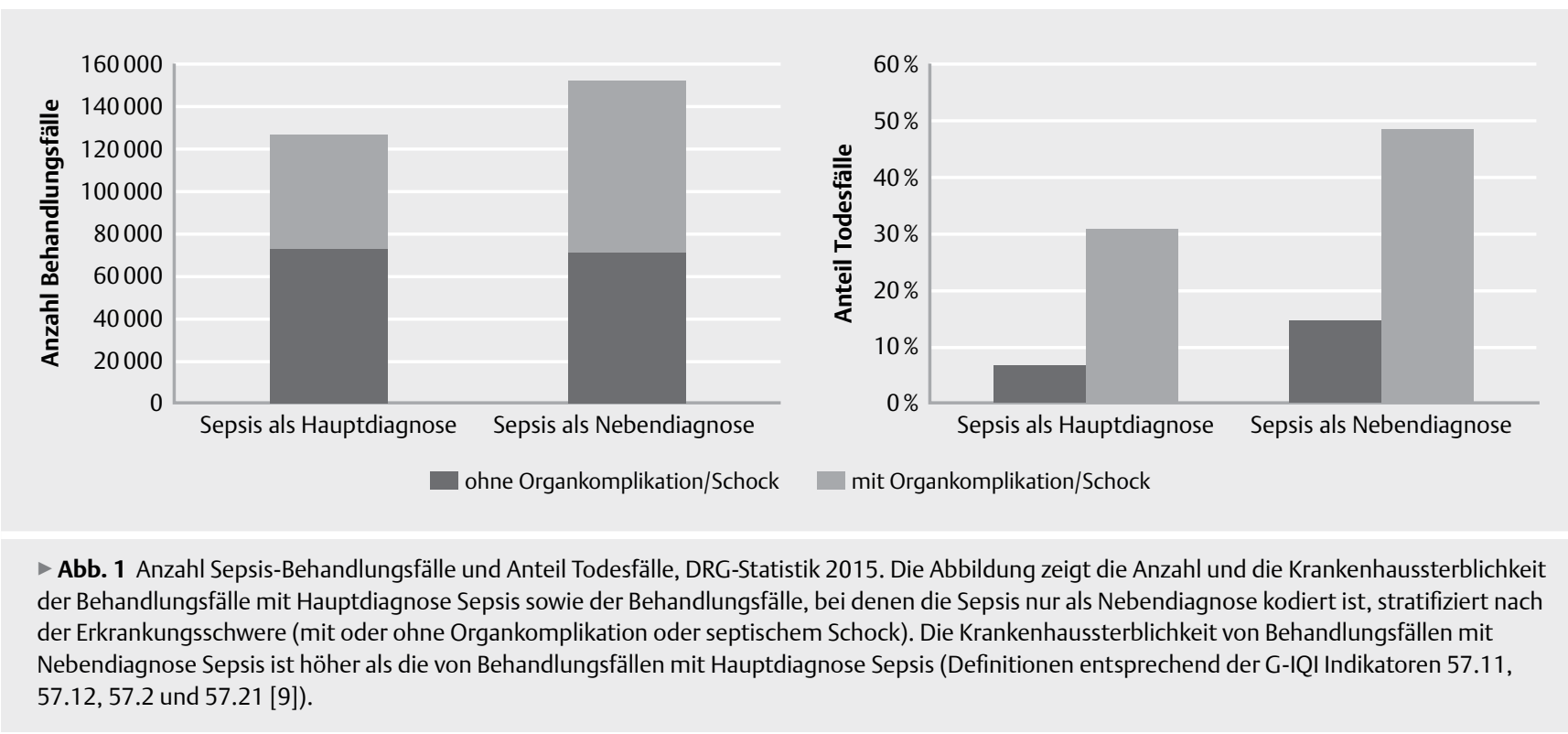




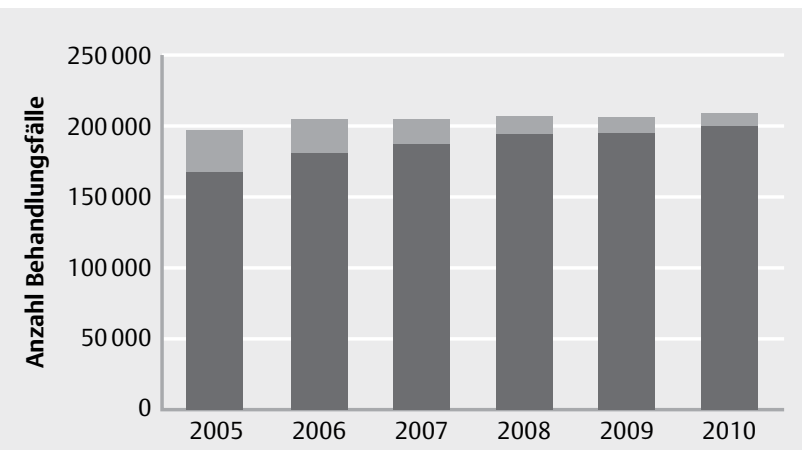

I63 Hirninfarkt I64 Schlaganfall, nicht näher bezeichnet

- Abb. 2 Anzahl Behandlungsfälle (ohne Zuverlegungen) mit den Hauptdiagnosen Hirninfarkt und Schlaganfall nicht näher bezeichnet, DRG-Statistik 2005 bis 2010. Die Abbildung zeigt die Entwicklung der Anzahl der Behandlungsfälle mit den Hauptdiagnosen 163 und 164 unter Ausschluss der aus einem anderen Krankenhaus zuverlegten Fälle. Die Betrachtung des ICD-Kodes I63 (Hirninfarkt) alleine könnte auf steigende Behandlungszahlen schließen lassen. Unter Mitberücksichtigung des unspezifischen ICD-Kodes I64 ergibt sich ein anderes Bild: Die Behandlungsfallzahlen bleiben zwischen 2006 und 2010 weitgehend unverändert, es findet lediglich eine Verschiebung hin zu einer höheren Kodiergenauigkeit statt [10]. zugefügt oder geändert wurden. Auch die Änderung von klinischen Krankheitsdefinitionen (z. B. Nicht-ST-Strecken-Hebungsinfarkt) kann die Anzahl der über Diagnosekodes identifizierten Behandlungsfälle im Zeitverlauf beeinflussen. Daneben ändert sich auch das Kodierverhalten der Krankenhäuser, was insbesondere in den frühen Jahren nach Einführung des DRG-Systems zu beobachten war. Deshalb kann es in zeitlichen Betrachtungen sinnvoll sein, die Definition der Analyseeinheiten etwas weiter zu fassen, um damit Veränderungen in der ICD-Klassifikation oder mögliche Veränderungen des Kodierverhaltens im Analysezeitraum aufzufangen (siehe Beispiel in > Abb. $2[10]$ ).

\section{Definition der Analyseeinheiten ausgehend von einer Behandlungsleistung}

Andere Fragestellungen der Versorgungsforschung beziehen sich primär auf Patienten, die eine bestimmte Art der Versorgung (z. B. eine Operation) erhalten haben. Behandlungsfälle mit einer bestimmten Behandlungsleistung können über die entsprechenden OPS-kodierten Prozedurenkodes identifiziert werden. Dazu muss zunächst festgelegt werden, welche Prozedurenkodes die interessierende Behandlung abbilden.

Die Systematik des OPS ist in einigen Abschnitten sehr komplex. So gibt es bspw. unterschiedliche OPS-Kodes für ähnliche Prozedu-

- Tab. 1 Mögliche OPS-Kodes zur Kodierung von Operationen an der Halsschlagader.

\begin{tabular}{|l|l|}
\hline OPS-Kode & Bezeichnung \\
\hline $5-380.0-$ & Inzision, Embolektomie und Thrombektomie von Blutgefäßen Arterien Kopf, extrakraniell, und Hals \\
\hline $5-381.0-$ & Endarteriektomie Arterien Kopf, extrakraniell, und Hals \\
\hline 5-382.0- & Resektion von Blutgefäßen mit Reanastomosierung Arterien Kopf, extrakraniell, und Hals \\
\hline $5-383.0-$ & Resektion und Ersatz (Interposition) von (Teilen von) Blutgefäßen Arterien Kopf, extrakraniell, und Hals \\
\hline 5-395.0- & Patchplastik an Blutgefäßen Arterien Kopf, extrakraniell, und Hals \\
\hline 5-397.0- & Andere plastische Rekonstruktion von Blutgefäßen Arterien Kopf, extrakraniell, und Hals \\
\hline
\end{tabular}

Die Tabelle zeigt verschiedene OPS-Kodes, die im Rahmen einer Operation einer verengten Halsschlagader kodiert werden könnten (entspricht der Definition des G-IQI Indikators 26.1 [9]).

> Tab. 2 Fallbeispiel: Prozedurenkodes, die im Rahmen einer einzigen Operation an der Wirbelsäule kodiert wurden.

\begin{tabular}{|l|l|}
\hline OPS-Kode & Bezeichnung \\
\hline $5-032.00$ & Zugang zur Lendenwirbelsäule, zum Os sacrum und zum Os coccygis, Zugang zur LWS, dorsal, 1 Segment \\
\hline $5-032.10$ & Zugang zur Lendenwirbelsäule, zum Os sacrum und zum Os coccygis, Flavektomie LWS, 1 Segment \\
\hline $5-831.0$ & Exzision von erkranktem Bandscheibengewebe, Exzision einer Bandscheibe \\
\hline $5-832.4$ & Exzision von erkranktem Knochen- und Gelenkgewebe der Wirbelsäule, Arthrektomie, partiell \\
\hline $5-83 b .50$ & Osteosynthese (dynamische Stabilisierung) an der Wirbelsäule, durch Schrauben-Stab-System, 1 Segment \\
\hline $5-83 b .70$ & Osteosynthese (dynamische Stabilisierung) an der Wirbelsäule, durch intervertebrale Cages, 1 Segment \\
\hline $5-835.9$ & Knochenersatz an der Wirbelsäule, Transplantation von Spongiosa(spänen) oder kortikospongiösen Spänen (autogen) \\
\hline $5-835 . c 0$ & Knochenersatz an der Wirbelsäule, Verwendung von humaner demineralisierter Knochenmatrix, ohne Anreicherung von Knochenwachstumszellen \\
\hline $5-839.60$ & Andere Operationen an der Wirbelsäule, knöcherne Dekompression des Spinalkanals, 1 Segment \\
\hline 5-837.6 & Wirbelkörperersatz und komplexe Rekonstruktion der Wirbelsäule, dorsoventrale Korrektur \\
\hline $\begin{array}{l}\text { Die Tabelle zeigt ein Fallbeispiel der dokumentierten Prozedurenkodes im Rahmen einer einzigen Operation an der Wirbelsäule. In der Wirbelsäulen- } \\
\text { chirurgie muss eine einzelne Operation häufig mit mehreren Prozedurenkodes dokumentiert werden, um alle Komponenten des Eingriffs darzustellen. }\end{array}$ \\
$\begin{array}{l}\text { Eine Zählung der Anzahl von Prozedurenkodes ohne Berücksichtigung der Fallebene kann zu Fehlinterpretationen führen, wenn jeder Kode als eigener } \\
\text { Eingriff gewertet wird. In diesem Beispiel würden dann fälschlicherweise zehn Eingriffe gezählt, obwohl alle angegebenen Prozeduren im Rahmen einer } \\
\text { einzigen Operation erbracht wurden [9]. }\end{array}$ \\
\hline
\end{tabular}


- Tab. 3 Mögliche OPS-Kodes zur Kodierung von kolorektalen Resektionen vor und nach 2013.

\begin{tabular}{|c|c|c|c|}
\hline \multicolumn{2}{|r|}{ Bis 2012} & \multicolumn{2}{|r|}{ Ab 2013} \\
\hline OPS-Kode & Bezeichnung & OPS-Kode & Bezeichnung \\
\hline 5-455.- & Partielle Resektion des Dickdarmes & 5-455.- & Partielle Resektion des Dickdarmes \\
\hline 5-456.- & (Totale) Kolektomie und Proktokolektomie & 5-456.- & (Totale) Kolektomie und Proktokolektomie \\
\hline 5-458.- & Erweiterte Kolonresektion mit Entfernung von Nachbarorganen & & \\
\hline 5-484.- & Rektumresektion unter Sphinktererhaltung & 5-484.- & Rektumresektion unter Sphinktererhaltung \\
\hline 5-485.- & Rektumresektion ohne Sphinktererhaltung & 5-485.- & Rektumresektion ohne Sphinktererhaltung \\
\hline \multicolumn{4}{|c|}{$\begin{array}{l}\text { Der OPS-Kode 5-458.- Erweiterte Kolonresektion mit Entfernung von Nachbarorganen war bis } 2012 \text { im OPS enthalten und wurde ab dem Jahr } 2013 \\
\text { ersatzlos gestrichen (da dieser Sachverhalt auch mit den anderen Kodes für Dickdarmresektion in Kombination mit Kodes für die Resektion anderer } \\
\text { Organe abgebildet werden kann). Um sowohl vor als auch nach der Änderung des OPS alle kolorektalen Resektionen zu erfassen, muss der Kode } \\
\text { 5-458.- in die Falldefinition mit einbezogen werden, da sonst die Fallzahlen in den Jahren vor } 2013 \text { unterschätzt würden. }\end{array}$} \\
\hline
\end{tabular}

- Tab. 4 Ein- und Ausschlusskriterien zur Abbildung planbarer offen-chirurgischer Eingriffe an der abdominellen Aorta bei Aneurysma.

\begin{tabular}{|c|c|c|}
\hline Merkmal & Einschluss & Ausschluss \\
\hline \multirow{2}{*}{$\begin{array}{l}\text { Haupt- oder } \\
\text { Nebendiagnose } \\
\text { (ICD-10-GM) }\end{array}$} & $\begin{array}{l}\text { I71.00 I71.01 I71.02 I71.03 171.2 I71.4 I71.6 I71.9 (Dissektion } \\
\text { oder Aneurysma an der Aorta, ohne Angabe einer Ruptur) }\end{array}$ & $\begin{array}{l}\text { I71.04 I71.05 I71.06 I71.07 I71.1 I71.3 I71.5 I71.8 (Dissektion oder } \\
\text { Aneurysma an der Aorta, rupturiert) }\end{array}$ \\
\hline & UND & ODER \\
\hline \multirow[t]{3}{*}{ Prozedur (OPS) } & $\begin{array}{l}\text { 5-382.33 5-382.3x 5-384.5 5-384.6 5-384.7 5-384.x } \\
\text { 5-384.y (Resektion mit Reanastomosierung oder Resektion } \\
\text { und Ersatz an der abdominellen Aorta) }\end{array}$ & $\begin{array}{l}\text { 5-382.30 5-382.32 5-384.0 5-384.1 5-384.2 5-384.3 5-384.4 } \\
\text { 5-384.8 5-38a.7 5-38a.8 5-38a.a 5-38a.b (Resektion mit Reanasto- } \\
\text { mosierung oder Resektion und Ersatz an der thorakalen Aorta) }\end{array}$ \\
\hline & & ODER \\
\hline & & $\begin{array}{l}\text { 5-38a.0 5-38a.1 5-38a.c (Endovaskuläre Implantation von } \\
\text { Stent-Prothesen abdominale Aorta oder Aorta nicht näher bezeichnet) }\end{array}$ \\
\hline \multicolumn{3}{|c|}{$\begin{array}{l}\text { Die Tabelle zeigt ein Beispiel für eine Definition der Analyseeinheiten anhand komplexer Merkmalskombinationen. Eingeschlossen werden Behand- } \\
\text { lungsfälle, bei denen ein nicht-rupturiertes Aneurysma (jeglicher Lokalisation) als Haupt- oder Nebendiagnose und ein Prozedurenkode für eine } \\
\text { offen-chirurgische Operation an der abdominellen Aorta dokumentiert ist. Fälle, in denen gleichzeitig ein rupturiertes Aneurysma als Diagnose oder } \\
\text { ein Prozedurenkode für eine Operation an der thorakalen Aorta oder für einen endovaskulären Eingriff an der abdominellen Aorta angegeben ist, } \\
\text { werden ausgeschlossen (entspricht der Definition des G-IQI Indikators } 27.21 \text { [9]). }\end{array}$} \\
\hline
\end{tabular}

ren, die manchmal auch an verschiedenen Stellen gelistet sind.

- Tab. 1 zeigt als Beispiel OPS-Kodes, die im Rahmen einer Operation einer verengten Halsschlagader kodiert werden könnten [9]. Da sich sowohl die bevorzugten Operationsverfahren als auch das Kodierverhalten zwischen Krankenhäusern unterscheiden können, sollten möglichst alle in Frage kommenden OPS-Kodes in die Definition eingeschlossen werden. Eine zu eng gefasste Definition kann ggf. zu einer Unterschätzung der Behandlungsfallzahlen führen, deren Ausmaß auf der Krankenhausebene variieren kann.

Häufig sind zu einem einzigen Behandlungsfall mehrere ähnliche Prozeduren dokumentiert, die jedoch nicht notwendigerweise mehrere Eingriffe darstellen. So wird bspw. in der Wirbelsäulenchirurgie eine einzelne Operation häufig mit mehreren Prozedurenkodes abgebildet, um alle Komponenten des Eingriffs darzustellen (siehe Fallbeispiel in > Tab. 2 [9]). Daher sollten Prozeduren immer fallbezogen gezählt werden, d. h. es werden Behandlungsfälle mit mindestens einem der interessierenden OPS-Kodes identifiziert. Eine Zählung der Prozedurenkodes ohne Berücksichtigung der Behandlungsfallebene kann zu Fehlinterpretationen führen, wenn jeder Kode als eigener Eingriff gewertet wird.

In den DRG-Daten sind ab dem Datenjahr 2006 zu jeder Prozedur Datum und Uhrzeit der Erbringung dokumentiert. Mit diesen Angaben könnten zeit- und datumsgleiche Prozedurenkodes einer Operation zugeordnet werden. Zur Verlässlichkeit der Zeit- und Da- tumsangaben (handelt es sich dabei wirklich um den Zeitpunkt der Erbringung oder eher um den Zeitpunkt der Dokumentation?) haben die Autoren bisher jedoch noch keine systematischen Erkenntnisse.

Der OPS unterliegt - in stärkerem Ausmaß als die ICD-10-GM einem stetigen Wandel. Aufgrund des medizinisch-technischen Fortschritts werden jedes Jahr neue OPS-Kodes in die Systematik eingefügt, mit denen neue Verfahren abgebildet werden. In der Vergangenheit wurden auch einige Abschnitte umstrukturiert, was mit Streichungen und Ersetzungen von Kodes einherging. Wenn sich die Analyse auf mehrere Datenjahre erstreckt, müssen mögliche Änderungen des OPS im Untersuchungszeitraum bei der Auswahl der relevanten OPS-Kodes berücksichtigt werden. Nur dann ist sichergestellt, dass sowohl vor als auch nach einer Änderung der jeweils gleiche Sachverhalt abgebildet wird (siehe Beispiel in \ Tab. 3). Wenn Änderungen in der Klassifikation nicht angemessen berücksichtigt werden, besteht die Gefahr, dass klassifikationsbedingte Artefakte als wahre Veränderungen fehlinterpretiert werden.

Definition der Analyseeinheiten anhand komplexer Merkmalskombinationen und Bildung von Untergruppen

Die Definition der Analyseeinheiten kann anhand von beliebigen Merkmalskombinationen erfolgen, die als Ein- oder Ausschlusskriterien festgelegt werden. Das Ziel ist meist die Bildung möglichst 
homogener Gruppen mit vergleichbarem Risikoprofil oder vergleichbaren Anforderungen an die Versorgung.

Häufig werden Kombinationen von Diagnosen und Prozeduren definiert, mit denen Behandlungsfälle mit einer bestimmten Versorgung im Zusammenhang mit einer bestimmten Erkrankung

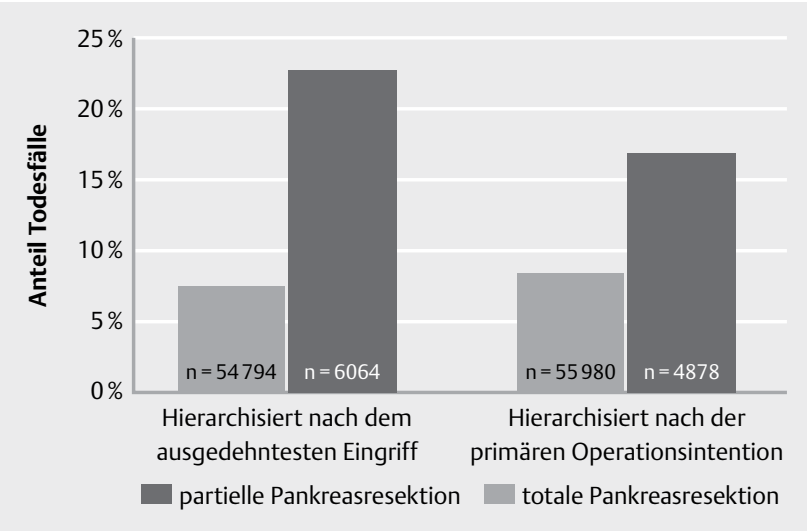

- Abb 3 Krankenhaussterblichkeit bei partieller und totaler Pankreasresektion in Abhängigkeit von der Hierarchisierung, DRG-Statistik 2009 bis 2014. Im Zeitraum von 2009 bis 2014 wurden 1.186 Behandlungsfälle identifiziert, bei denen sowohl eine partielle als auch eine totale Pankreasresektion dokumentiert waren. Die Krankenhaussterblichkeit bei diesen Behandlungsfällen liegt bei $48 \%$. Je nachdem, wie diese Behandlungsfälle in der Hierarchisierung den Gruppen zugeteilt werden, ergeben sich unterschiedliche Krankenhaussterblichkeiten [11]. (z. B. Dickdarmresektion bei Kolonkarzinom) identifiziert werden sollen. In diesen Fällen können die Einschlussdiagnosen (z. B. in Bezug auf die Unterscheidung von Haupt- oder Nebendiagnose oder bezüglich der Lokalisation einer Erkrankung) etwas weiter gefasst werden, da die Analyseeinheiten zusätzlich über eine spezifische Prozedur definiert sind. $>$ Tab. 4 zeigt als Beispiel die Definition zur Abbildung planbarer offen-chirurgischer Eingriffe an der abdominellen Aorta bei Aneurysma, die im Indikatorensystem der German Inpatient Quality Indicators (G-IQI) verwendet wird [9]. Die G-IQI Definitionen, die zahlreiche Krankheitsbilder und Behandlungen abbilden, können ein Ausgangspunkt für die Definition solch komplexer Falldefinitionen sein.

Analyseeinheiten werden zur Stratifizierung häufig in Untergruppen eingeteilt. Um zu vermeiden, dass Behandlungsfälle in mehreren Untergruppen gezählt werden, muss die Einteilung hierarchisch erfolgen, d. h. jeder Behandlungsfall darf nur einer Untergruppe zugeordnet werden. Dabei muss entschieden werden, wie einzelne Merkmale in der Hierarchie eingeordnet werden. So können bspw. Behandlungsfälle mit Resektion der Bauchspeicheldrüse (Pankreas) danach unterteilt werden, ob eine partielle oder eine totale Resektion durchgeführt wurde. In ca. $2 \%$ aller Behandlungsfälle mit Pankreasresektion ist jedoch sowohl eine partielle als auch eine totale Resektion dokumentiert, da nach einer partiellen Pankreasresektion bei einem komplizierten postoperativen Verlauf die vollständige Entfernung der Bauchspeicheldrüse im gleichen stationären Aufenthalt notwendig werden kann. Wenn nun in der Hierarchie die totale Resektion vorrangig gezählt wird, werden die Gruppen nach dem jeweils ausgedehntesten dokumentierten Ein-

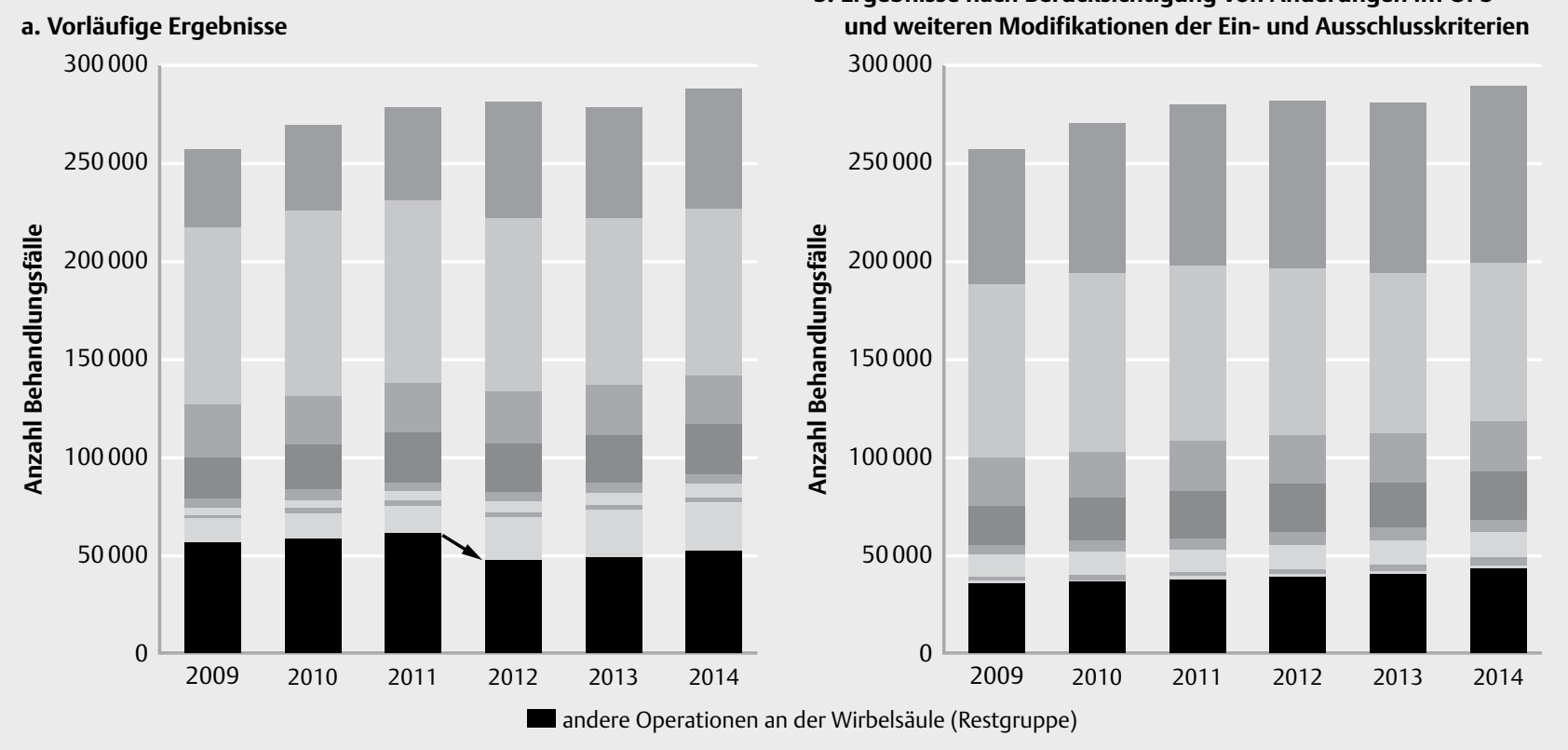

- Abb. 4 Zeitliche Betrachtung von Wirbelsäulenoperationen stratifiziert nach Untergruppen, DRG-Statistik 2009 bis 2014. In den vorläufigen Ergebnissen a ist zwischen den Jahren 2011 und 2012 ein plötzlicher Rückgang der Fallzahl in der Gruppe „Andere Operation an der Wirbelsäule“ erkennbar. Dies resultiert daraus, dass in den Ein- und Ausschlusskriterien zur Einteilung in Untergruppen OPS-Kodes einbezogen wurden, die erst ab 2012 kodierbar waren. In den Jahren davor werden Behandlungsfälle, die über diese OPS-Kodes definiert sind, der Restgruppe „Andere Operationen an der Wirbelsäule“ zugeordnet. In der endgültigen Analyse b wurden die Änderungen im OPS bei der Definition von Untergruppen berücksichtigt und weitere Modifikationen der Ein- und Ausschlusskriterien vorgenommen [12]. 
griff unterteilt. Wenn die partielle Resektion vorrangig gezählt wird, bezieht sich die Einteilung eher auf die primäre Operationsintention (siehe $>$ Abb. 3 [11]).

Je mehr Merkmale in der Falldefinition berücksichtigt werden, umso komplexer kann sich die Hierarchisierung gestalten. Wenn die Analyse einen längeren Zeitraum betrachtet, müssen alle Merkmale, die zur Definition der Analyseeinheiten herangezogen werden, auf mögliche Änderungen in der Klassifikation überprüft werden. Hierzu sind häufig Voranalysen nötig, wobei eine grafische Darstellung helfen kann, verdächtige Brüche in der Entwicklung zu entdecken (siehe Beispiel in \ Abb. 4 [12]).

\section{Definition der Zielgrößen: Was soll gemessen werden?}

Neben deskriptiven Analysen von Behandlungsfallzahlen haben Fragestellungen der Versorgungsforschung häufig die Bewertung der Versorgungssituation zum Ziel. Die Zielgrößen dafür können entsprechend dem Throughput-Modell der Versorgungsforschung auf verschiedenen Ebenen analysiert werden [13]. So können einerseits die erbrachten Versorgungsleistungen als Zielgrößen definiert werden, die bspw. abbilden, wie viele Behandlungsfälle eine angemessene Versorgung erhalten haben (Output). In anderen Fragestellungen kann das patienten- oder bevölkerungsbezogene Behandlungsergebnis (Outcome) von Interesse sein.

Bei allen Arten von Zielgrößen muss berücksichtigt werden, dass in Krankenhausabrechnungsdaten die Beobachtungszeit für einen Behandlungsfall auf den stationären Aufenthalt begrenzt ist. Damit kommen als Zielgrößen nur solche Versorgungsleistungen oder Ergebnisse in Frage, die während des stationären Aufenthaltes erbracht werden bzw. eintreten können.

\section{Erbrachte Versorgungsleistungen (Output)}

Je nach Fragestellungen können unterschiedliche Versorgungsleistungen als Zielgrößen definiert werden. Dabei muss beachtet werden, dass nicht alle Leistungen in Krankenhausabrechnungsdaten verlässlich dokumentiert sind. So ist bei nicht-entgeltrelevanten diagnostischen Leistungen (z. B. Ultraschalluntersuchungen) von einer tendenziell unvollständigen Erfassung auszugehen. Geeignetere Zielgrößen sind solche Versorgungsleistungen, bei denen aufgrund der Entgeltrelevanz für die Krankenhäuser eine vollständige Dokumentation wahrscheinlich ist (z. B. Linksherzkatheterintervention bei Behandlungsfällen mit Hauptdiagnose Herzinfarkt).

Die erbrachten Versorgungsleistungen werden häufig zwischen Leistungserbringern oder Regionen verglichen, um die Qualität der Versorgung zu bewerten. Wenn die Analyseeinheiten als homogene Gruppe mit vergleichbaren Anforderungen an die Versorgung definiert sind, dann ist zur Bewertung der Versorgung häufig keine Risikoadjustierung erforderlich.

\section{Behandlungsergebnis (Outcome)}

Ein typischer Outcome-Parameter in fallbezogenen Krankenhausabrechnungsdaten ist die Krankenhaussterblichkeit, die über den Entlassungsgrund „Tod“ operationalisiert wird [8]. Es handelt sich dabei um eine verlässlich messbare Zielgröße, da Todesfälle in Krankenhausabrechnungsdaten eindeutig erfasst werden. Beachtet werden muss, dass hierbei Todesfälle aus allen Gründen (death from all causes) betrachtet werden. Eine eindeutige Zuordnung der Todesfälle zu einem potentiell verursachenden Ereignis bzw. einer Grunderkrankung ist in der Regel nicht möglich [14].

Eine mögliche weitere Zielgröße ist das Auftreten unerwünschter Ereignisse, die häufig als Komplikationen einer Behandlung definiert sind und über bestimmte Nebendiagnosen oder Prozeduren identifiziert werden können. Dabei muss berücksichtigt werden, dass für klinisch und abrechnungstechnisch nicht oder wenig relevante Zustände mit einer Untererfassung zu rechnen ist. So werden bspw. erfolgreich behandelte katheterassoziierte Harnwegsinfekte, die keinen wesentlichen Einfluss auf den klinischen Verlauf hatten, bei schweren Grunderkrankungen vermutlich eher unterkodiert sein [14].

In einigen Fällen kann es sinnvoll sein, Komplikationen nicht über Nebendiagnosen, sondern über damit zusammenhängende, entgeltrelevante Prozedurenkodes zu identifizieren. Damit werden schwere, behandlungsbedürftige Komplikationen erfasst, während solche ohne Behandlungsbedarf unberücksichtigt bleiben. Als Beispiel seien Blutungskomplikationen im Zusammenhang mit Operationen oder invasiven Eingriffen genannt. Diese lassen sich mit ICD-Kodes abbilden (z. B. T81.0, D62, R58), die jedoch bezüglich der Schwere der Blutung nicht spezifisch sind. Auch die Vollständigkeit der Dokumentation ist unsicher und auf der Krankenhausebene möglicherweise vom Kodierverhalten abhängig. Dagegen ist es aber relativ wahrscheinlich, dass die Gabe von Bluttransfusionen, für die ab sechs Transfusionseinheiten ein Zusatzentgelt abgerechnet werden kann, von den Krankenhäusern vollständig dokumentiert wird. Werden anstelle von Behandlungsfällen mit Blutungsdiagnosen solche mit Prozedurenkodes für die Gabe von sechs oder mehr Transfusionseinheiten identifiziert, werden damit schwere Blutungskomplikationen relativ verlässlich und vollständig abgebildet.

In den deutschen Krankenhausabrechnungsdaten gibt es kein Datenfeld zur Unterscheidung, ob eine Nebendiagnose bereits bei Aufnahme bestanden hat oder erst während des Krankenhausaufenthaltes aufgetreten ist. Vor dem Hintergrund der Fragestellung und der Definition der Analyseeinheiten muss abgewogen werden, wie wahrscheinlich es ist, dass eine Nebendiagnose eine Komplikation darstellt. In Zusammenhang mit planbaren Eingriffen (z. B. Hüftgelenkprothesenimplantation bei Arthrose) können bestimmte Nebendiagnosen, wie akuter Herzinfarkt, Lungenembolie, Lungenentzündung oder Sepsis, mit hoher Wahrscheinlichkeit als Komplikation gewertet werden.

Ob die Operationalisierung von Komplikationen sachgerecht ist, kann über die Krankenhaussterblichkeit der Behandlungsfälle mit identifizierter Komplikation abgeschätzt werden. Bei schweren Komplikationen würde man eine vergleichsweise höhere Krankenhaussterblichkeit erwarten (siehe Beispiel in > Abb. 5 [15]). Darüber hinaus kann die Krankenhaussterblichkeit von Behandlungsfällen mit Komplikation als eigener Ergebnisparameter definiert und vergleichend gemessen werden. Die Sterblichkeit von Behandlungsfällen, bei denen eine Komplikationen dokumentiert ist (failure to rescue), lässt - insbesondere im Zusammenhang mit komplexen operativen Eingriffen - Rückschlüsse auf das Komplikationsmanagement von Krankenhäusern zu [16]. 


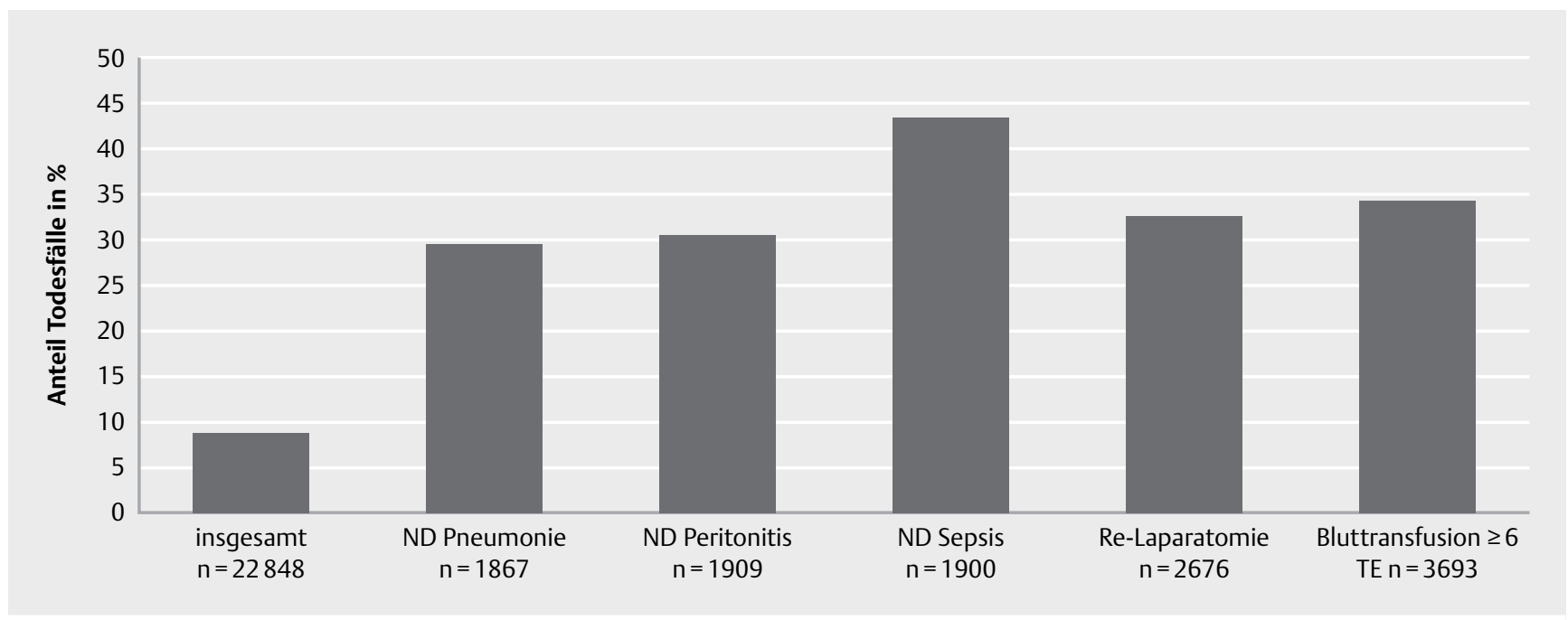

Abb 5 Krankenhaussterblichkeit bei Pankreasoperationen wegen bösartiger Neubildung, insgesamt und bei Komplikationen, DRG-Statistik 2009 bis 2013. Die Abbildung zeigt die Krankenhaussterblichkeit von Behandlungsfällen mit Pankreasoperation wegen bösartiger Neubildung der Bauchspeicheldrüse. Die Sterblichkeit liegt insgesamt bei 8,6\%. Bei Behandlungsfällen, bei denen Komplikationen als Nebendiagnose oder Prozeduren, die auf Komplikationen schließen lassen, dokumentiert sind, ist die Krankenhaussterblichkeit deutlich erhöht [15]. ND: Nebendiagnose. TE: Transfusionseinheit.

\section{Risikoadjustierung: Wie können mögliche Verzerrungen kontrolliert werden?}

Behandlungsergebnisse werden in der Versorgungsforschung häufig vergleichend untersucht, um auf Determinanten der Versorgungsqualität und/oder mögliche Verbesserungspotentiale zu schließen. So können Outcomes bspw. zwischen Regionen, in Abhängigkeit von der Art der Versorgung oder in Abhängigkeit von Merkmalen der Leistungserbringer verglichen werden. Auch im Zeitverlauf können Behandlungsergebnisse vergleichend untersucht werden, bspw. vor und nach einer Intervention.

Unterschiedliche Risikoverteilungen in den Vergleichsgruppen können zu Verzerrungen führen, da die beobachteten OutcomeUnterschiede möglicherweise nicht durch die interessierende Variable, sondern durch den Einfluss ungleich verteilter Störgrößen bedingt sind. Zweck der Risikoadjustierung ist, den Einfluss der interessierenden Variable auf das Outcome unabhängig von (messbaren) Störgrößen darzustellen. Die Herausforderung besteht darin, Störgrößen anhand der in den DRG-Daten verfügbaren Informationen verlässlich abzubilden.

Häufig relevante Risikofaktoren auf der Ebene der Behandlungsfälle sind das Alter und das Geschlecht der behandelten Patienten. In Krankenhausabrechnungsdaten ist von einer weitgehend vollständigen und auch korrekten Erfassung dieser Merkmale auszugehen, da die Plausibilität von Alter und Geschlecht im Zusammenhang mit bestimmten Diagnosen und Prozeduren bei der Eingruppierung in eine DRG-Fallpauschale geprüft wird. Bei unplausiblen Konstellationen (z. B. Prostataoperationen bei Frauen) kann kein Entgelt abgerechnet werden.

Eine weitere mögliche Einflussgröße für das Behandlungsergebnis ist der Schweregrad einer Erkrankung. In der ICD-10-GM ist dieser häufig nur eingeschränkt darstellbar. So ist bspw. keine Unterscheidung der TNM-Stadien bei Tumorerkrankungen möglich. Aber auch dort, wo Unterteilungen nach dem Schweregrad einer Erkran- kung kodierbar sind, ist nicht immer von einer vollständigen Dokumentation auszugehen. Beispielsweise ist in der ICD bei der chronischen obstruktiven Lungenerkrankung (COPD) eine Unterteilung nach dem Grad der Obstruktion vorgesehen. Im Jahr 2015 wurde jedoch bei mehr als $30 \%$ der Behandlungsfälle mit der Hauptdiagnose COPD der Grad der Obstruktion als nicht näher bezeichnet angegeben (G-IQI Indikator 15.16 [9]).

In manchen Fällen lässt sich der Schweregrad einer Erkrankung indirekt einschätzen. Beispielsweise kann die Ausdehnung einer soliden Tumorerkrankung über die Ausdehnung des zugehörigen operativen Eingriffs approximiert werden. Ein transapikaler Zugang bei der minimalinvasiven Implantation einer Aortenklappe könnte auf eine schwerwiegende Atherosklerose der peripheren Gefäße hinweisen. Es sei jedoch angemerkt, dass der Einbezug von Prozeduren in die Risikoadjustierung bei Fragestellungen, die auf die Behandlungsqualität von Krankenhäusern abzielen, auch problematisch sein kann. Dies gilt dann, wenn die Wahl der angemessen Operationsmethode als Bestandteil der Behandlungsqualität gewertet werden kann, wie bspw. die Wahl des operativen Zugangs (laparoskopisch oder offen chirurgisch) bei Gallenblasenentfernungen.

Auch Begleiterkrankungen, die neben der Grunderkrankung bestehen, können das Behandlungsergebnis beeinflussen. In Krankenhausabrechnungsdaten können Begleiterkrankungen über kodierte Nebendiagnosen identifiziert werden. Hier besteht die Herausforderung darin, vorbestehende Begleiterkrankungen von Komplikationen zu unterscheiden. Wenn die Fragestellung die Behandlungsqualität zum Gegenstand hat, ist eine Adjustierung nach (potentiellen) Komplikationen nicht zielführend, da damit mögliche Qualitätsunterschiede „wegadjustiert“ würden [14]. Bei chronischen Erkrankungen wie z. B. Herzinsuffizienz, chronische Lungenerkrankung oder Diabetes ist es jedoch in den meisten Fällen sehr wahrscheinlich, dass diese bereits vor dem stationären Aufenthalt bestanden haben. 


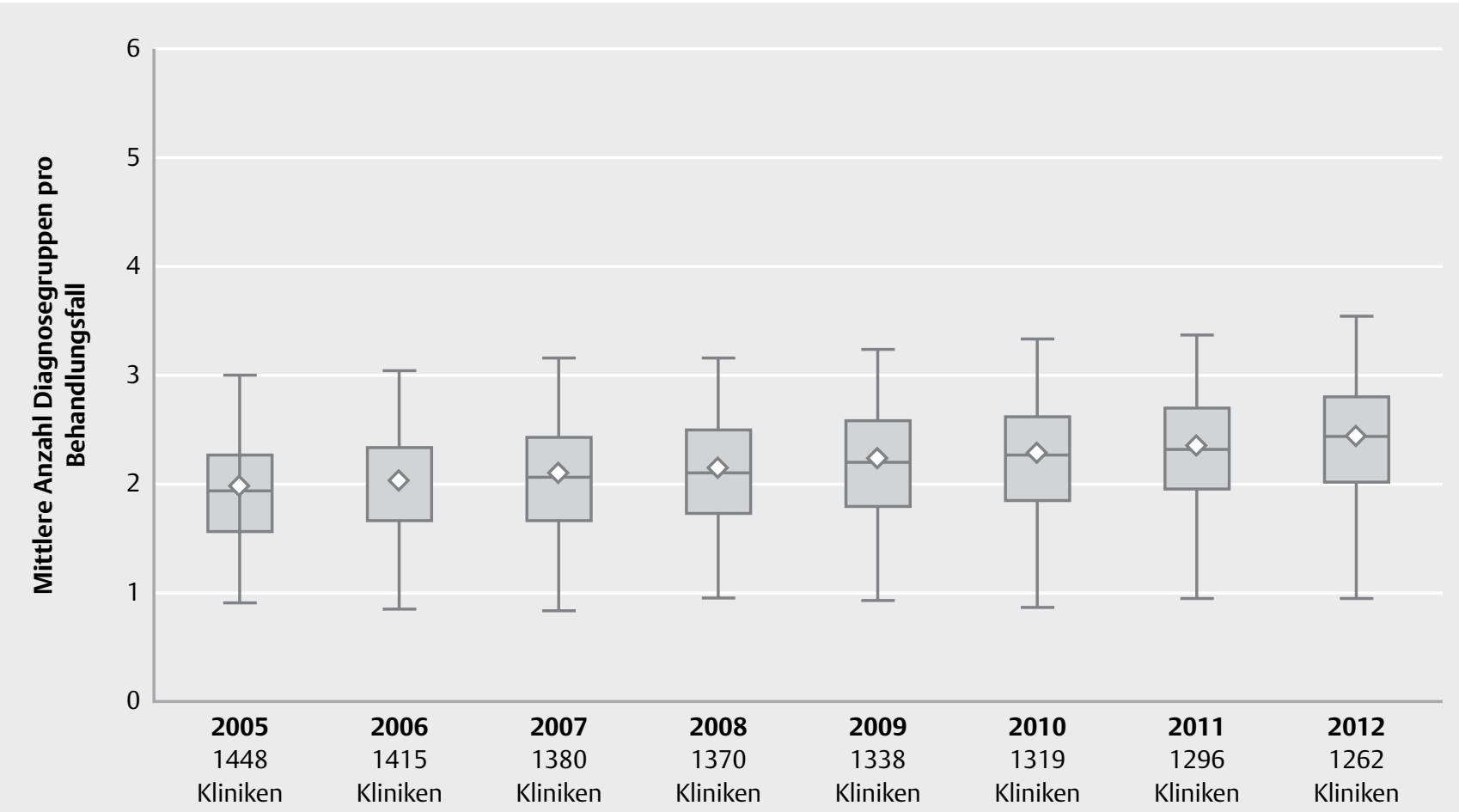

- Abb 6 Mittlere Anzahl von Elixhauser-Diagnosegruppen pro Behandlungsfall bei Behandlungsfällen mit Hauptdiagnose Hüftgelenknahe Fraktur: Verteilung über die Krankenhäuser, DRG-Statistik 2005 bis 2012. Für jeden Behandlungsfall wurde anhand der dokumentierten Nebendiagnosen die Anzahl der Elixhauser-Diagnosegruppen [21] bestimmt. Für jedes Krankenhaus wurde der alters- und geschlechtsstandardisierte Mittelwert der Diagnosegruppen pro Behandlungsfall berechnet. Die Verteilung der Mittelwerte über die Krankenhäuser ist als Boxplot dargestellt. Die vertikalen Linien erstrecken sich von 5. bis zum 95. Perzentil. Zwischen 2005 und 2012 ist eine zunehmende Variation der Kodierung zwischen den Krankenhäusern erkennbar [22].

Auf Diagnosen basierende Risikoindizes z. B. nach Charlson [17] oder Elixhauser [18] können einen Ausgangspunkt für die Definition von Variablen zur Risikoadjustierung bieten. Solche Indizes können jedoch problematisch sein, wenn sie unkritisch eingesetzt werden [19]. So ist es bei einigen der enthaltenen Diagnosen möglich, dass diese - je nachdem welche Analyseeinheiten betrachtet werden eher Komplikationen der Behandlung als Begleiterkrankungen darstellen. Der Elixhauser Index enthält z. B. in den neueren Definitionen [20, 21, 23] die Diagnose Lungenembolie, die im Falle von Operationen eine potentiell vermeidbare Komplikation darstellt. Der Charlson-Index, der ursprünglich zur Vorhersage der 1-Jahres-Sterblichkeit nach Krankenhausaufenthalten entwickelt wurde, beinhaltet akute Zustände, die in vielen Fragestellungen als Komplikationen gewertet werden müssen (z. B. akuter Herzinfarkt, Schlaganfall). Bei der Verwendung von Risikoindizes müssen daher in Abhängigkeit von der Fragestellung alle enthaltenen Diagnosen inhaltlich auf ihre Eignung geprüft werden, damit die Diagnosegruppen gezielt ausgewählt und ggf. angepasst werden können. Je nach Untersuchungsgegenstand werden jedoch möglicherweise nicht alle relevanten Risikofaktoren in solchen Indizes erfasst. Daher bevorzugen die Autoren eine spezifisch auf den Untersuchungsgegenstand ausgerichtete, literaturgestützte Auswahl von Faktoren für die Risikoadjustierung mit anschließender Operationalisierung unter Berücksichtigung der Verlässlichkeit der verwendeten Datenfelder.
Wenn längere Zeiträume in die Analyse einbezogen werden, müssen auch mögliche zeitliche Veränderungen bei der Kodierung von Nebendiagnosen beachtet werden. So hat bspw. in den Jahren nach der DRG-Einführung die Vollständigkeit der Nebendiagnosenkodierung zugenommen. Dies bedeutet jedoch auch, dass in frühen Betrachtungsjahren von einer Untererfassung auszugehen ist, die auf der Krankenhausebene variieren kann. Insbesondere bei nicht entgeltrelevanten Diagnosen oder Prozeduren kann ein unterschiedliches Kodierverhalten in Bezug auf die Vollständigkeit der Dokumentation angenommen werden. > Abb. $\mathbf{6}$ zeigt ein Beispiel für die Variation der Kodierung von Nebendiagnosen zwischen Krankenhäusern [22]. Wenn die Definition der Risikoadjustierungsvariablen sehr anfällig gegenüber dem Kodierverhalten verschiedener Krankenhäuser ist, würde im Extremfall nicht nach der ggf. unterschiedlichen Fallzusammensetzung, sondern nach dem Kodierverhalten der Leistungserbringer adjustiert [14, 24].

\section{Organisationsbezogene Fragestellungen: Wie können Merkmale der Krankenhäuser identifiziert werden?}

Bestimmte Fragestellungen der Versorgungsforschung, wie z. B. die Umsetzung der Mindestmengenregelungen, die Verbreitung bestimmter Behandlungsangebote oder die Analyse von Zusammenhängen zwischen Merkmalen der Krankenhäuser und Behand- 
lungsergebnissen, erfordern krankenhausbezogene Auswertungen. Dafür müssen die Datensätze einzelner Behandlungsfälle dem behandelnden Krankenhaus zugeordnet werden.

Krankenhäuser können in den DRG-Daten über das anonymisierte Institutionskennzeichen abgegrenzt werden, das zu jedem Behandlungsfall angegeben ist. Hierbei ist zu beachten, dass das Institutionskennzeichen primär eine abrechnende Einheit repräsentiert und damit nicht unbedingt gleichbedeutend mit einem Krankenhaus bzw. Krankenhausstandort sein muss. So ist es z. B. möglich, dass mehrere Krankenhausstandorte unter einem gemeinsamen Institutionskennzeichen abrechnen. In der Querschnittbetrachtung führt dies zu einer Unterschätzung der Anzahl der Krankenhäuser bei gleichzeitiger Überschätzung der Behandlungsfallzahl pro Krankenhaus. Dies muss bei der Interpretation der Ergebnisse berücksichtigt werden. Im Zeitverlauf können sich sowohl die Institutionskennzeichen an sich als auch die Anzahl der darunter abrechnenden Kliniken (z. B. im Rahmen von Fusionen) ändern. Dies beeinträchtigt die Nachverfolgbarkeit von Krankenhauskohorten [25]. In solchen Fällen empfiehlt es sich Restgruppen zu bilden, um den hiervon betroffenen Anteil der Fälle darzustellen und den möglichen Einfluss auf die Ergebnisse bewerten zu können [26].

Neben dem Institutionskennzeichen steht in den DRG-Daten ein Standortkennzeichen zur Verfügung, das bislang jedoch nur dann verwendet wird, wenn die Standorte einer differenzierten Festlegung des Versorgungsauftrages im Krankenhausplan unterliegen. Hier muss auch beachtet werden, dass das Standortkennzeichen derzeit nur den Standort bei Entlassung kennzeichnet. Bei internen Verlegungen entspricht der entlassende Standort möglicherweise nicht dem Erbringungsort der interessierenden Behandlungsleistung (z. B. einer Operation).

In den Mikrodaten der DRG-Statistik sind bezogen auf die Krankenhäuser nur wenige Informationen verfügbar. Neben Institutions- und Standortkennzeichen sind nur Regionalinformationen zum Krankenhausstandort enthalten. Weitere krankenhausbezogene Informationen lassen sich jedoch auch direkt aus dem Datenbestand ableiten. So kann z. B. das Leistungsvolumen eines Krankenhauses bestimmt werden. Hierfür wird die Anzahl bestimmter Behandlungsfälle in einem Zeitraum pro Institutionskennzeichen ausgezählt. Diese Information wird dann den Behandlungsfällen zugespielt, um diese nach der Leistungsmenge ihres behandelnden Krankenhauses einzuteilen. Entsprechend lassen sich auch Informationen über ein bestimmtes Leistungsangebot ableiten. So kann bspw. eine bestimmte Anzahl von dokumentierten neurologischen Komplexbehandlungen in einem Krankenhaus auf das Vorhandensein einer Einheit zur spezialisierten Schlaganfallversorgung schließen lassen [26].

Da die Forschungsdatenzentren bei der Bereitstellung von Mikrodaten zur wissenschaftlichen Nutzung den Anforderungen des Bundesstatistikgesetzes unterliegen, dürfen einzelne Krankenhäuser in den Daten nicht identifiziert werden. Es dürfen aber auch keine anonymisierten Ergebnisse einzelner Krankenhäuser (z. B. in Form von Funnelplots) ausgegeben werden. Ergebnisse auf der Krankenhausebene müssen immer für Gruppen von Krankenhäusern zusammengefasst werden [3].

\section{FAZIT}

Die Vorteile der DRG-Daten liegen in der Vollständigkeit, dem Bevölkerungsbezug und den einheitlichen Regeln für die Datendokumentation. Die Verlässlichkeit und Interpretierbarkeit von Analysen auf der Grundlage dieser Daten hängt jedoch entscheidend von der sachgerechten Operationalisierung der Variablen ab. Um das Risiko von Verzerrungen und Fehlinterpretationen möglichst gering zu halten, sind umfangreiche Vorüberlegungen notwendig, die neben medizinisch-klinischen Aspekten auch den Kontext der Datenerhebung und die klassifikatorischen Möglichkeiten zur Abbildung bestimmter Sachverhalte berücksichtigen müssen. Man muss die richtigen Fragen an die Daten stellen, nur dann erhält man auch richtige, d. h. verlässlich interpretierbare Antworten. Eine Orientierung an bestehenden Standards zur Analyse von Routinedaten [27] und den Empfehlungen zur Publikation solcher Analysen [28, 29] ist uneingeschränkt zu empfehlen.

Es ist zu hoffen, dass die Akzeptanz von Studien auf der Grundlage von Krankenhausabrechnungsdaten in Zukunft weiter zunimmt und der wertvolle Datenbestand der DRGDaten noch intensiver für Forschungszwecke genutzt wird.

\section{Interessenkonflikt}

Das Fachgebiet Strukturentwicklung und Qualitätsmanagement im Gesundheitswesen an der Technischen Universität Berlin, in dem Melissa Spoden von 2016 bis 2018 tätig war, und in dem Ulrike Nimptsch und Thomas Mansky von 2010 bis 2018 tätig waren, war eine Stiftungsprofessur der Helios Kliniken $\mathrm{GmbH}$.

\section{Literatur}

[1] Deutsche Krankenhausgesellschaft (DKG). GKV-Spitzenverband, Verband der privaten Krankenversicherung (PKV), Institut für das Entgeltsystem im Krankenhaus (InEK GmbH). Deutsche Kodierrichtlinien. Allgemeine und Speziell Kodierrichtlinien für die Verschlüsselung von Krankheiten und Prozeduren. Version 2018. Düsseldorf: Deutsche Krankenhaus Verlagsgesellschaft 2017

[2] Busley A. Den Blick auf Qualität und Kosten. MDK forum 2018; 4: 12-13. https://www.mdk.de/aktuelles-presse/magazin-mdk-forum/ mdk-forum-42018/ (Zugriff am 25.04.2019)

[3] Nimptsch U, Schömann M, Mansky T. Datenzugang für die Qualitätsmessung in der akutstationären Krankenhausversorgung. In: Dormann F, Klauber J, Kuhlen R, Hrsg. Qualitätsmonitor 2019. Berlin: Medizinisch Wissenschaftliche Verlagsgesellschaft; 2018 (im Druck)

[4] Forschungsdatenzentren der Statistischen Ämter des Bundes und der Länder. Datenangebot | Fallpauschalenbezogene Krankenhausstatistik (DRG-Statistik).Wiesbaden: Statistisches Bundesamt; 2018: http:// www.forschungsdatenzentren.de/bestand/drg/index.asp (Zugriff am 31.10.2018)

[5] Grobe TG, Nimptsch U, Friedrich J. Krankenhausbehandlung. In: Swart E, Ihle P, Gothe H, Matusiewicz D, Hrsg. Routinedaten im Gesundheitswesen. Handbuch Sekundärdatenanalyse: Grundlagen, Methoden und Perspektiven. Bern: Verlag Hans Huber; 2014: 121-145 
[6] Forschungsdatenzentren der Statistischen Ämter des Bundes und der Länder. Metadatenreport. Teil II: Produktspezifische Informationen zur Nutzung der Fallpauschalenbezogenen Krankenhausstatistik 2016 für die Kontrollierte Datenfernverarbeitung (EVAS-Nummer: 23141). Version 1. doi:10.21242/23141.2016.00.00.1.1.Wiesbaden: Statistische Ämter des Bundes und der Länder 2019

[7] Statistisches Bundesamt. Fallpauschalenbezogene Krankenhausstatistik (DRG-Statistik). Qualitätsbericht 2017. Wiesbaden: Statistisches Bundesamt 2018; https://www.destatis.de/DE/Publikationen/ Qualitaetsberichte/Gesundheitswesen/FallpauschaleKrankenhausstatistk.pdf?_blob = publicationFile (Zugriff am 31.10.2018)

[8] Nimptsch U, Bestmann A, Erhart M et al. Zugang zu Routinedaten. In: Swart E, Ihle P, Gothe H, Matusiewicz D, Hrsg. Routinedaten im Gesundheitswesen. Handbuch Sekundärdatenanalyse: Grundlagen, Methoden und Perspektiven. Bern: Verlag Hans Huber; 2014: 270-290

[9] Mansky T, Nimptsch U, Cools A et al. G-IQI | German Inpatient Quality Indicators. Version 5.1. - Band 2: Definitionshandbuch für das Datenjahr 2017. Berlin: Universitätsverlag der TU Berlin; 2017, doi:10.14279/depositonce-6070

[10] Nimptsch U, Mansky T. Trends in der akutstationären Schlaganfallversorgung in Deutschland. Dtsch Arztebl Int 2012; 109: 885-892

[11] Krautz C, Nimptsch U, Weber GF et al. Effect of Hospital Volume on In-hospital Morbidity and Mortality Following Pancreatic Surgery in Germany. Ann Surg 2018; 267: 411-417

[12] Nimptsch U, Bolczek C, Spoden M et al. Mengenentwicklung stationärer Behandlungen bei Erkrankungen der Wirbelsäule - Analyse der deutschlandweiten Krankenhausabrechnungsdaten von 2005 bis 2014. Z Orthop Unfall 2018; 156: 175-183

[13] Schrappe M, Pfaff H. Versorgungsforschung vor neuen Herausforderungen: Konsequenzen für Definition und Konzept. Gesundheitswesen 2016; 78: 689-694

[14] Mansky T, Nimptsch U. Qualitätsmessung im Krankenhaus - worauf kommt es an? Z Evid Fortbild Qual Gesundhwes 2014; 108: 487-494

[15] Nimptsch U, Krautz C, Weber GF et al. Nationwide In-hospital Mortality Following Pancreatic Surgery in Germany is Higher than Anticipated. Ann Surg 2016; 264: 1082-1090

[16] Silber JH, Romano PS, Rosen AK et al. Failure-to-rescue: comparing definitions to measure quality of care. Med Care 2007; 45: 918-925

[17] Charlson ME, Pompei P, Ales KL et al. A new method of classifiying prognostic comorbidity in longitudinal studies: development and validation. Journal of Chronic Diseases 1987; 40: 373-383
[18] Elixhauser A, Steiner C, Harris DR et al. Comorbidity measures for use with administrative data. Med Care 1998; 36: 8-27

[19] Lux G, Biermann J, Dahl H et al. Risikoadjustierung und Komorbiditäten. In: Swart E, Ihle P, Gothe H, Matusiewicz D, Hrsg. Routinedaten im Gesundheitswesen. 2. Auflage. Bern: Hans Huber; 2014: 411-423

[20] Agency for Healthcare Research and Quality (AHRQ). Healthcare Cost and Utilization Project (HCUP). Comorbidity Software, Version 3.7. AHRQ: Rockville 2018 Abrufbar unter http://www.hcup-us.ahrq.gov/ toolssoftware/comorbidity/comorbidity.jsp (Zugriff am 31.10.2018)

[21] Turner K, Burchill C. Elixhauser Comorbidity Index macro code using ICD-10.University of Manitoba; Winnipeg: 2006Abrufbar unter http:// mchp-appserv.cpe.umanitoba.ca/Upload/SAS/_ElixhauserICD10.sas. txt (Zugriff am 31.10.2018)

[22] Nimptsch U. Disease-Specific Trends of Comorbidity Coding and Implications for Risk Adjustment in Hospital Administrative Data. Health Services Research 2016; 51: 981-1001

[23] Quan H, Sundararajan V, Halfon P et al. Coding algorithms for defining comorbidities in ICD-9-CM and ICD-10 administrative data. Med Care 2005; 43: 1130-1139

[24] Nicholl J. Case-mix adjustment in non-randomised observational evaluations: the constant risk fallacy. J Epidemiol Community Health 2007; 61: 1010-1013

[25] Nimptsch U, Wengler A, Mansky T. Kontinuität der Institutionskennzeichen in Krankenhausabrechnungsdaten - Analyse der bundesweiten DRG-Statistik von 2005 bis 2013. Z Evid Fortbild Qual Gesundhwes 2016; 117: 38-44

[26] Nimptsch U, Mansky T. Stroke unit care and trends of in-hospital mortality for stroke in Germany 2005-2010. Int J Stroke 2014; 9: 260-265

[27] Swart E, Gothe H, Geyer S et al. Gute Praxis Sekundärdatenanalyse (GPS): Leitlinien und Empfehlungen. 3. Fassung; Version 2012/2014. Ge-sundheitswesen 2015; 77: 120-126

[28] Swart E, Bitzer EM, Gothe $\mathrm{H}$ et al. STandardisierte BerichtsROutine für Sekundärdaten Analysen (STROSA) - ein konsentierter Berichtsstandard für Deutschland, Version 2. Gesundheitswesen 2016; 78 (S 01): e145-e160

[29] Benchimol El, Smeeth L, Guttmann A et al. RECORD Working Committee The REporting of studies Conducted using Observational Routinely-collected health Data (RECORD) statement. PLoS Med 2015; 12: e1001885 